\title{
Correction to: Minimally conscious state "plus": diagnostic criteria and relation to functional recovery
}

\author{
Aurore Thibaut $^{1,5}$ (1) $\cdot$ Yelena G. Bodien ${ }^{2,3} \cdot$ Steven Laureys ${ }^{1,5} \cdot$ Joseph T. Giacino ${ }^{3,4}$
}

Published online: 19 March 2020

(c) Springer-Verlag GmbH Germany, part of Springer Nature 2020

\section{Correction to: Journal of Neurology https://doi.org/10.1007/s00415-019-09628-y}

Following electronic publication of the above-referenced manuscript, we discovered that one of the three criteria we proposed to establish command-following in the MCS+syndrome was inadvertently omitted in some parts of the manuscript. Specifically, "object recognition" was omitted from the criteria used to diagnose MCS+. We operationally define command-following as a Coma Recovery Scale-Revised (CRS-R) Auditory Subscale score of 3 (i.e. reproducible command-following: A3) or 4 (i.e. consistent commandfollowing: A4), or a Visual Subscale score of 5 (i.e. object recognition: V5). However, in Tables 1 and 2 and Figs. 2 and 3, we failed to include object recognition as a criterion for MCS+. In addition, in Fig. 4 (CRS-R Face Sheet), object recognition is not labeled as a criterion for MCS+. Therefore, we would like to highlight the corrected parts of our manuscript to clarify that the criteria for "command-following" include object recognition (V5) as well as reproducible (A3) and consistent (A4) command-following.

The original article can be found online at https://doi.org/10.1007/ s00415-019-09628-y.

Aurore Thibaut

athibaut@ulg.ac.be

1 Coma Science Group, GIGA-Consciousness, University of Liège and University Hospital of Liège, Allée de l'hopital, 11-B34, 4000 Liège, Belgium

2 Department of Neurology, Massachusetts General Hospital, Center for Neurotechnology and Neurorecovery, Harvard Medical School, Boston, MA, USA

3 Department of Physical Medicine and Rehabilitation, Spaulding Rehabilitation Hospital, Harvard Medical School, Boston, MA, USA

4 Department of Psychiatry, Massachusetts General Hospital, Boston, USA

5 Centre du Cerveau ${ }^{2}$, University Hospital of Liège, Liège, Belgium
Please note that these modifications do not change any of the findings of the study.

Please find the individual corrections here:

In the section "Methods" the paragraph "consistent command following" needs to be replaced by:

Command following: At least three clearly-discernible behavioral responses are observed over four consecutive trials on at least one of two commands. Note that this definition also includes object recognition.

At the end of the "Procedures" paragraph, the following text needs to be added before Table 1 .

Note that we did not include "attention" from the Arousal Subscale as a criterion for MCS+ because all responses, regardless of accuracy, are considered when scoring this item. This item can be scored even in the absence of intact language function. For example, a patient with aphasia who does not comprehend language but understands that a response is anticipated may meet the required criteria.

At the end of the section "Results" the word "lower" needs to be replaced by "higher":

The MCS - group had significantly higher (i.e., worse) DRS scores than all other groups (all $p$ values $<0.05$ - supplementary Fig. S3).

Please find the corrected Figs. 2, 3 and 4, as well as supplemental Fig. 3, on the following page: 
Fig. 2 DRS total scores (means and SEs) for each group at transition from UWS to MCS(black column) or at transition from UWS or MCS - to $\mathrm{MCS}+$ (six grey columns). $C F$ command following; IC intentional communication; $I V$ intelligible verbalization; DRS Disability Rating Scale, $M C S$ - minimally conscious state minus; Black asterisks represent statistical differences between groups corrected for multiple comparisons (Bonferroni corrected). Grey asterisks represent a significant difference uncorrected for multiple comparisons
Fig. 3 DRS total scores (means and SEs) for each group at discharge from rehabilitation. $C F$ command following; IC intentional communication; $I V$ intelligible verbalization; $D R S$ Disability Rating Scale, MCSminimally conscious state minus; Black asterisks represent statistical differences between groups corrected for multiple comparisons (Bonferroni corrected). Grey asterisks represent a significant difference uncorrected for multiple comparisons
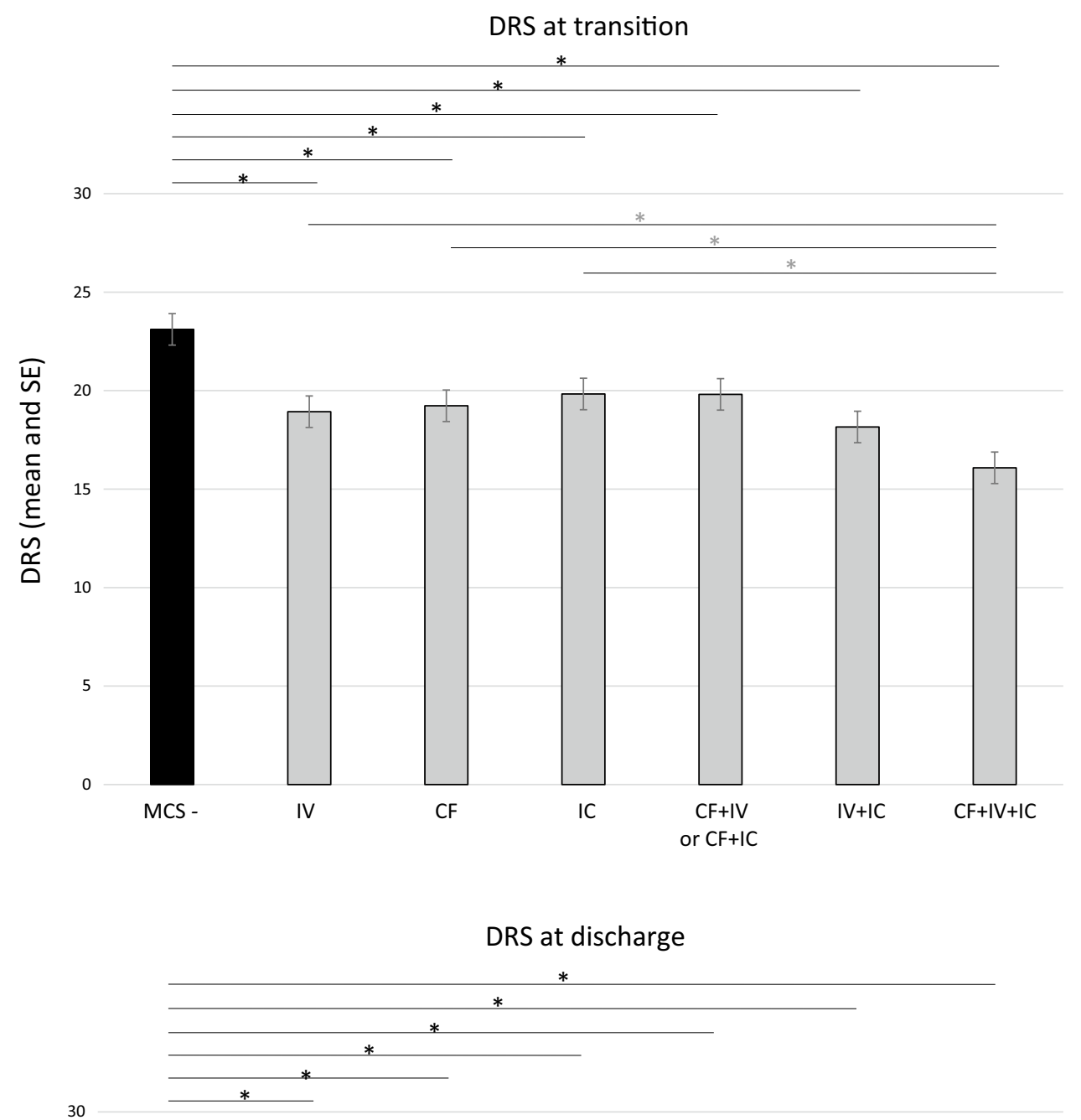

25

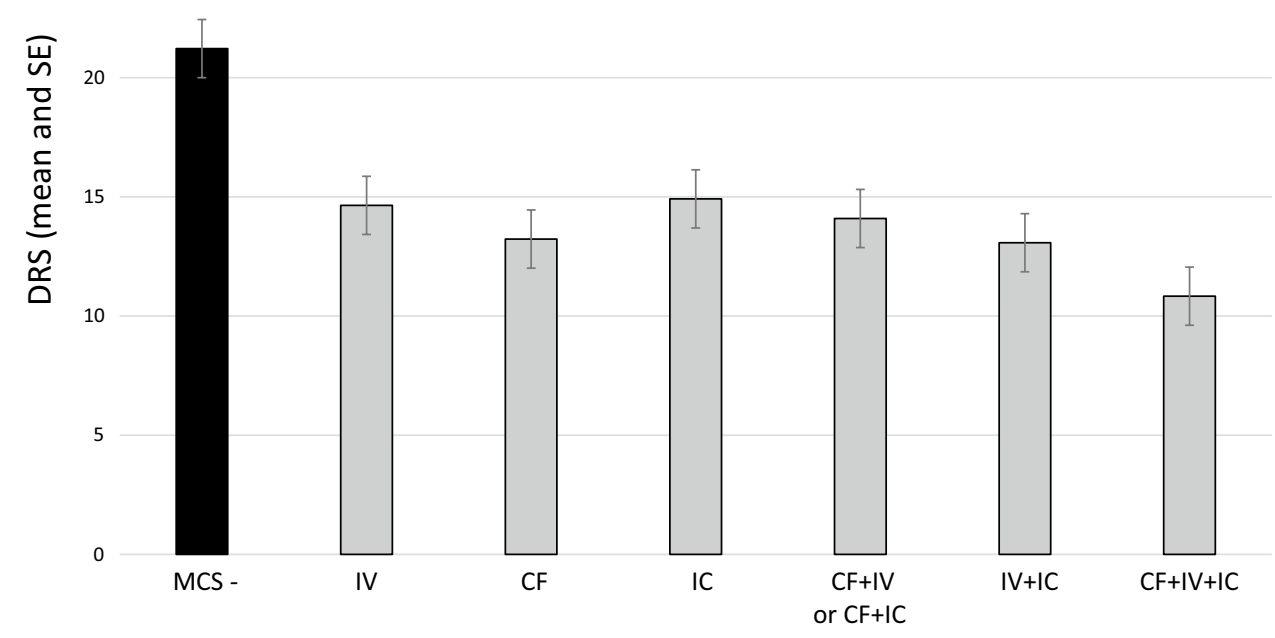




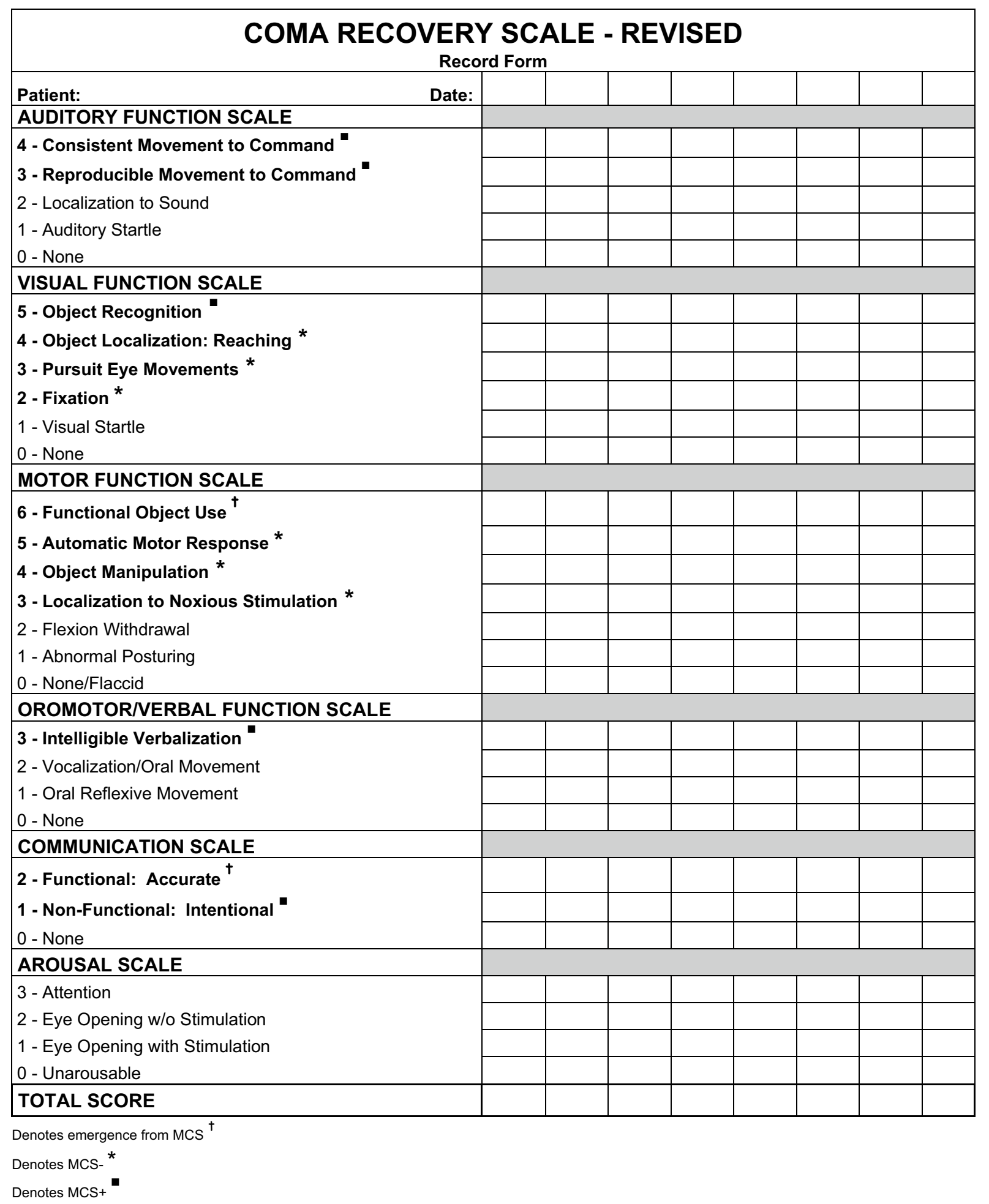

Fig. 4 Coma recovery scale-revised (CRS-R) record form showing behavioral criteria for MCS-, MCS+ and MCS emergence 
Supplementary Fig. 3 DRS without the communication subscale at time of transition. DRS total scores removing the communication subscale (means and SEs) for each group at transition from UWS to MCS- (black column) or at transition from UWS or MCSto MCS+ (six grey columns). $\mathrm{CF}=$ command following; IC = intentional communication; IV = intelligible verbalization; DRS $=$ Disability Rating Scale, MCS- = minimally conscious state minus. Black asterisks represent statistical differences between groups
DRS w/o communication subscale at time of transition
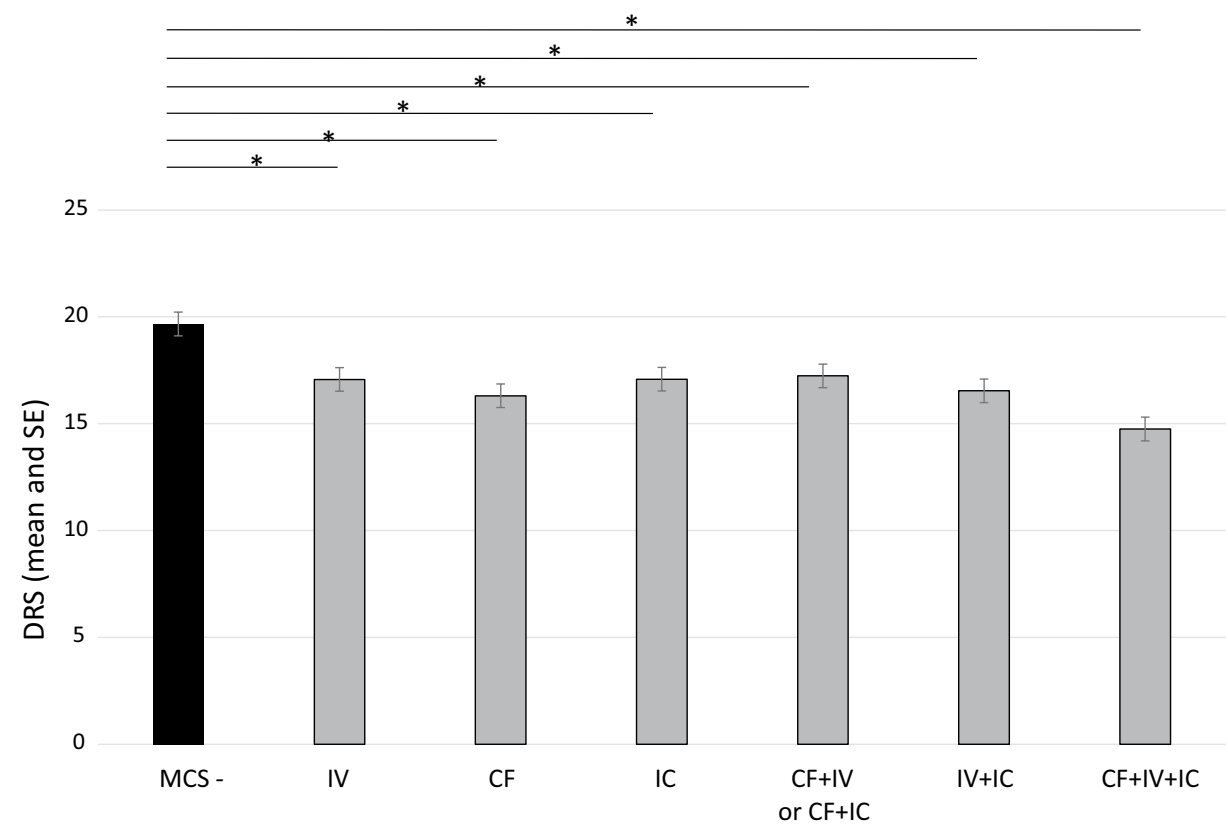

Please find the corrected Tables 1 and 2 below:

Table 1 Operational criteria for minimally conscious state (MCS) plus and minus

\begin{tabular}{|c|c|}
\hline Group & Operational criteria \\
\hline 1. MCS plus-command following only & $\begin{array}{l}\text { Score of } 3 \text { or } 4 \text { on the auditory subscale or a score of } 5 \text { on the visual } \\
\text { subscale }\end{array}$ \\
\hline 2. MCS plus-intelligible verbalization only & Score of 3 on the CRS-R verbal subscale \\
\hline 3. MCS plus-intentional communication only & Score of 1 on the CRS-R communication subscale \\
\hline $\begin{array}{l}\text { 4. MCS plus-command-following and intentional communication or } \\
\text { intelligible verbalization }\end{array}$ & $\begin{array}{l}\text { Score of } 3 \text { or } 4 \text { on the auditory subscale or a score of } 5 \text { on the visual } \\
\text { subscale and a score of } 1 \text { on the communication subscale or a score } \\
\text { of } 3 \text { on the verbal subscale }\end{array}$ \\
\hline 5. MCS plus-intelligible verbalization and intentional communication & $\begin{array}{l}\text { Score of } 3 \text { on the verbal subscale and score of } 1 \text { on the communication } \\
\text { subscale }\end{array}$ \\
\hline $\begin{array}{l}\text { 6. MCS plus-command following and intelligible verbalization and } \\
\text { intentional communication }\end{array}$ & $\begin{array}{l}\text { Score of } 3 \text { or } 4 \text { on the auditory subscale or a score of } 5 \text { on the visual } \\
\text { subscale and a score of } 3 \text { on the verbal subscale and a score of } 1 \text { on } \\
\text { the communication subscale }\end{array}$ \\
\hline 7. MCS minus & $\begin{array}{l}\text { No evidence of language function but demonstrates at least one } \\
\text { behavioral feature of MCS (i.e., visual pursuit, visual fixation, object } \\
\text { localization, localization to noxious stimulation, object manipulation, } \\
\text { automatic motor behavior) }\end{array}$ \\
\hline
\end{tabular}


Table 2 Demographic characteristics and DRS scores at admission, time of transition (from UWS to MCS-/+or from MCS- to MCS+) and discharge

\begin{tabular}{|c|c|c|c|c|c|c|c|c|c|}
\hline Group & $N$ & $\begin{array}{l}\text { Diagnosis at } \\
\text { admission/ } \\
\text { etiology }\end{array}$ & $\begin{array}{l}\text { Age (gender- } \\
\text { female) }\end{array}$ & $\begin{array}{l}\text { DRS at } \\
\text { admission } \\
\text { (severity } \\
\text { category) }\end{array}$ & $\begin{array}{l}\text { Time at } \\
\text { admission } \\
\text { (days post } \\
\text { injury) }\end{array}$ & $\begin{array}{l}\text { DRS at time } \\
\text { of transition } \\
\text { to MCS } \\
\text { (severity } \\
\text { category) }\end{array}$ & $\begin{array}{l}\text { Days post } \\
\text { injury at tran- } \\
\text { sition from } \\
\text { UWS to MCS } \\
\text { or MCS- to } \\
\text { MCS+ }\end{array}$ & $\begin{array}{l}\text { DRS at } \\
\text { discharge } \\
\text { (severity } \\
\text { category) }\end{array}$ & $\begin{array}{l}\text { Days post injury } \\
\text { at discharge }\end{array}$ \\
\hline All & 120 & $\begin{array}{l}57 \text { MCS-63 } \\
\text { UWS } \\
68 \text { TBI-52 } \\
\text { NTBI }\end{array}$ & $\begin{array}{l}46.68 \pm 18.85 \\
\text { (46 women) }\end{array}$ & $\begin{array}{l}21.83 \pm 2.79 \\
\text { (extremely } \\
\text { severe) }\end{array}$ & $32.23 \pm 18.98$ & $\begin{array}{l}19.21 \pm 3.21 \\
\text { (extremely } \\
\text { severe) }\end{array}$ & $48.20 \pm 25.95$ & $\begin{array}{l}14.06 \pm 5.07 \\
\text { (severe) }\end{array}$ & $119.02 \pm 78.88$ \\
\hline $\mathrm{CF}$ & 39 & $\begin{array}{l}18 \text { MCS-21 } \\
\text { UWS } \\
25 \text { TBI-14 } \\
\text { NTBI }\end{array}$ & $\begin{array}{l}38 \pm 17 \text { years } \\
\text { (16 women) }\end{array}$ & $\begin{array}{l}22.56 \pm 2.58 \\
\text { (vegetative } \\
\text { state) }\end{array}$ & $43.85 \pm 13.42$ & $\begin{array}{l}19.23 \pm 3.13 \\
\text { (extremely } \\
\text { severe) }\end{array}$ & $43.28 \pm 13.43$ & $\begin{array}{l}13.23 \pm 5.36 \\
\text { (severe) }\end{array}$ & $118.615 \pm 82.73$ \\
\hline IV & 14 & $\begin{array}{l}9 \text { MCS-5 } \\
\text { UWS } \\
7 \text { TBI-7 } \\
\text { NTBI }\end{array}$ & $\begin{array}{l}52 \pm 17 \text { years } \\
(6 \text { women })\end{array}$ & $\begin{array}{l}21.36 \pm 3.18 \\
\text { (extremely } \\
\text { severe) }\end{array}$ & $34.29 \pm 31.54$ & $\begin{array}{l}18.93 \pm 3.49 \\
\text { (extremely } \\
\text { severe) }\end{array}$ & $59.00 \pm 43.30$ & $\begin{array}{l}14.64 \pm 6.38 \\
\text { (severe) }\end{array}$ & $157.71 \pm 113.123$ \\
\hline IC & 12 & $\begin{array}{l}4 \text { MCS-8 } \\
\text { UWS } \\
6 \text { TBI-7 } \\
\text { NTBI }\end{array}$ & $\begin{array}{l}45 \pm 24 \text { years } \\
\text { ( } 7 \text { women) }\end{array}$ & $\begin{array}{l}22.00 \pm 2.26 \\
\text { (vegetative } \\
\text { state) }\end{array}$ & $41.59 \pm 32.65$ & $\begin{array}{l}19.83 \pm 2.16 \\
\text { (extremely } \\
\text { severe) }\end{array}$ & $59.58 \pm 37.55$ & $\begin{array}{l}14.92 \pm 4.72 \\
\text { (severe) }\end{array}$ & $145.58 \pm 92.59$ \\
\hline $\begin{array}{l}\mathrm{CF}+\mathrm{IV} \text { or } \\
\mathrm{IC}\end{array}$ & 21 & $\begin{array}{l}9 \text { MCS-12 } \\
\text { UWS } \\
15 \text { TBI-6 } \\
\text { NTBI }\end{array}$ & $\begin{array}{l}54 \pm 21 \text { years } \\
(5 \text { women })\end{array}$ & $\begin{array}{l}21.57 \pm 3.20 \\
\text { (extremely } \\
\text { severe) }\end{array}$ & $26.29 \pm 22.57$ & $\begin{array}{l}19.81 \pm 3.30 \\
\text { (extremely } \\
\text { severe) }\end{array}$ & $42.57 \pm 23.35$ & $\begin{array}{l}14.09 \pm 3.78 \\
\text { (severe) }\end{array}$ & $118.95 \pm 82.60$ \\
\hline $\mathrm{IV}+\mathrm{IC}$ & 13 & $\begin{array}{l}7 \text { MCS-6 } \\
\text { UWS } \\
4 \text { TBI-9 } \\
\text { NTBI }\end{array}$ & $\begin{array}{l}51 \pm 16 \text { years } \\
(6 \text { women })\end{array}$ & $\begin{array}{l}20.80 \pm 2.80 \\
\text { (extremely } \\
\text { severe) }\end{array}$ & $38.78 \pm 19.34$ & $\begin{array}{l}18.15 \pm 2.64 \\
\text { (extremely } \\
\text { severe) }\end{array}$ & $45.92 \pm 18.48$ & $\begin{array}{l}13.08 \pm 3.97 \\
\text { (severe) }\end{array}$ & $85.85 \pm 27.51$ \\
\hline $\mathrm{CF}+\mathrm{IV}+\mathrm{IC}$ & 12 & $\begin{array}{l}9 \text { MCS-3 } \\
\text { UWS } \\
7 \text { TBI-5 } \\
\text { NTBI }\end{array}$ & $\begin{array}{l}54 \pm 10(4 \\
\text { women) }\end{array}$ & $\begin{array}{l}19.92 \pm 2.31 \\
\text { (extremely } \\
\text { severe) }\end{array}$ & $30.08 \pm 13.52$ & $\begin{array}{l}16.08 \pm 2.27 \\
\text { (severe) }\end{array}$ & $41.42 \pm 14.59$ & $\begin{array}{l}10.83 \pm 3.24 \\
\text { (moderately } \\
\text { severe) }\end{array}$ & $98.67 \pm 36.77$ \\
\hline MCS- & 9 & $\begin{array}{c}2 \text { MCS-7 } \\
\text { UWS } \\
3 \text { TBI-6 } \\
\text { NTBI }\end{array}$ & $\begin{array}{l}43 \pm 19 \text { years } \\
(2 \text { women })\end{array}$ & $\begin{array}{l}23.89 \pm 1.26 \\
\text { (vegetative } \\
\text { state) }\end{array}$ & $41.33 \pm 16.53$ & $\begin{array}{l}23.11 \pm 0.53 \\
\text { (extremely } \\
\text { severe) }\end{array}$ & $63.00 \pm 32.88$ & $\begin{array}{l}21.22 \pm 1.72 \\
\text { (extremely } \\
\text { severe) }\end{array}$ & $100.33 \pm 31.67$ \\
\hline
\end{tabular}

DRS Disability Rating Scale; $M C S$ minimally conscious state; $U W S$ unresponsive wakefulness syndrome; $C F$ command following; $I V$ intelligible verbalization; IC intentional communication; TBI traumatic brain injury; NTBI non-traumatic brain injury; "+" two or more items are observed 